\title{
Anti-cancer effect of Scutellaria baicalensis in combination with cisplatin in human ovarian cancer cell
}

\author{
Bo Yoon Choi ${ }^{1}$, Jong Cheon Joo ${ }^{2}$, Yeon Kyu Lee ${ }^{1}$, Ik-Soon Jang ${ }^{3}$, Soo Jung Park ${ }^{4 *}$ and Yoon Jung Park ${ }^{1 *}$ (D)
}

\begin{abstract}
Background: Ovarian cancer is one of the major causes of death among females in worldwide. Cisplatin is a primary anti-cancer drug against ovarian cancer, but the recurrent tumors after treatment frequently show acquired chemoresistance. Extract of Scutellaria baicalensis (SbE) has been reported to have functional compounds including baicalin, which has anti-cancer effects. However, the anti-cancer effects of SbE in ovarian cancer and its underlying mechanisms are elusive.
\end{abstract}

Methods: We investigated that the effects of SbE and/or cisplatin on cell death in the cisplatin sensitive ovarian cancer cell line A2780 (CSC) and the counterpart cell line that has cisplatin resistance (CRC). Molecular mechanisms of the effects, focusing on apoptosis and autophagy, were examined.

Results: Treatment of cisplatin or SbE reduced cell viability significantly in CSC and too much lesser extent in CRC. Cisplatin-induced cell death in CSC was mediated by p53-induced apoptosis acompanied by expresson of damageregulated autophagy modulator (DRAM). In CRC, decreased DRAM expression $(p<0.01)$ hindered p21-mediated cell death and contributed to cisplatin resistance. Treatment of SbE also induced cell death in CSC by p53-dependent apoptosis, not in CRC. Autophagy was not induced by neither cisplatin nor SbE. Intriguingly, the combinational treatment of SbE and cisplatin significantly decreased cell viability in CRC. The cell death was mediated by autophagy with increased expression of Atg5 and Atg12 ( $p<0.05)$, rather than p53-dependent pathway with repressed expression of $p 21(p<0.001)$ through HDAC1 activation.

Conclusions: The combined treatment of SbE with cisplatin was effective in CRC, leading to cell death via Beclin1independent autophagy, suggesting that SbE treatment in combination with cisplatin has a potential as a chemotherapeutic agent in cisplatin-resistant ovarian cancer.

Keywords: Cell death, Drug resistance, Epigenomics, Herbal medicine, Ovary Neoplasms

\section{Background}

Ovarian cancer has remained one of common and lethal gynecological cancers for women in worldwide [1]. About the $70 \%$ of ovarian cancers are diagnosed at advanced stage [2] and a five-year survival rate was recorded about the $40 \%$ in all cancer staging of ovarian cancer [3]. Although therapeutic methods against cancer such as chemotherapy and

\footnotetext{
* Correspondence: soojungpark@woosuk.ac.kr; park.yoonjung@ewha.ac.kr ${ }^{4}$ Department of Sasang Constitutional Medicine, College of Korean Medicine, Woosuk University, Jeonju, Republic of Korea

${ }^{1}$ Department of Nutritional Science and Food Management, College of Science \& Industry Convergence, Ewha Womans University, Seoul, Republic of Korea

Full list of author information is available at the end of the article
}

surgery have rapidly developed in the past decades, identification of successful treatments against the ovarian cancer has been challenged due to its high rate for late-stage diagnosis and acquisition of drug resistance.

Cisplatin is a commonly used drug in the treatment of ovarian cancer, but it often faces the challenge of chemoresistance after repeated treatments, resulting in limiting drug effectiveness [4]. The resistance can be caused by multiple mechanisms, including inadequate cisplatin accumulation, cisplatin inactivation, enhanced DNA repair, and activation of survival signaling pathways [5]. Previous studies have found that expression level of the genes, related to intracellular drug 
accumulation, drug inactivation, DNA repair system, and survival signaling pathway, was significantly different between CSC and CRC [6-9].

Drug resistant cancer cells show uncontrolled cell proliferation and reduced cell death such as apoptosis and autophagy not responding to the treatment [10]. Apoptosis is a programmed process of unnecessary or dysfunctional cell death via DNA fragmentation, nuclear fragmentation, chromatin condensation, membrane blebbing and cell shrinkage [11]. p53 is a key modulator of cellular stress responses. p53 triggers apoptosis through up-regulating target genes in many cell types including cancer cells [12]. Target genes induced by p53 include the cyclin-dependent kinase inhibitor $p 21$ gene and the pro-apoptotic $B a x$ gene leading to apoptosis [13-15]. DRAM gene is another target gene, which is an important component of p53-induced apoptosis and triggers autophagy [16]. Autophagy is an intracellular self degradative process that dismantles unnecessary or dysfunctional cytoplasmic components and organelles in the lysosome. In cancer cells, some of anti-cancer therapeutic agents promote autophagy-induced cell death [17]. Autophagic pathway occurs through the formation of double membrane vesicle called autophagosome that encloses cytoplasmic components and organelles and then autophagosome transfers to lysosome for degradation [17]. Autophagosome formation involves multiple factors such as Beclin 1, autophagy-related protein (Atg)12-Atg5, and microtubule-associated protein light chain 3 (LC3) complexes [18]. The transfer to lysosome also requires DRAM in its membrane [19].

Extract of Scutellaria baicalensis (SbE) is an herbal medicine that have been used for anti-oxidant and antiinflammatory activities [20]. It is known to have multiple functional compounds including baicalin and baicalein. Baicalin is a flavone glycoside that has been reported to have anti-cancer effects in breast cancer and prostate cancer [21, 22]. Although baicalin as a single compound has been studied for its anti-cancer properties, few studies are available for anti-cancer effects of the extract [23]. In this study, we investigated whether SbE contributed to overcome cisplatin resistance using a cisplatinresistant ovarian cancer cell model and its possible mechanisms.

\section{Methods}

\section{Preparation of SbE}

Lyophilized SbE was obtained from Hanpoong Pham \& Foods Co., Ltd. (Jeonju, Korea). $300 \mathrm{~g} \mathrm{SbE}$ was refluxed for $3 \mathrm{~h}$ in $3 \mathrm{~L}$ of $30 \%$ ethanol, passed through $1 \mu \mathrm{m}$ filter, evaporated, and dried in vacuum less than $60{ }^{\circ} \mathrm{C}$ and pulverized. SbE, obtained with $115.3 \mathrm{~g}$ (38.43\% yield), was dissolved in dimethyl sulfoxide (DMSO) to make stock solutions of $250 \mathrm{mg} / \mathrm{mL}$ and then was diluted with serum- free RPMI 1640 for the working concentrations $(100 \sim 400 \mu \mathrm{g} / \mathrm{mL})$, resulting in the percentage of DMSO to dissolve the extract was less than $0.16 \%$, in final. Equal amounts of DMSO were included in controls.

\section{Liquid chromatography-mass spectrometer (LC-MS) analysis}

A liquid chromatograpy mass spectroscopy (LC-MS) analysis was achieved using an Agilent 6410B triple quadrupole (Agilent Technologies, Wilmington, DE, USA) equipped with electrospray ionization (ESI) (Agilent Technologies, Wilmington, DE, USA), according to a manufacturer's protocol. Briefly, $100 \mathrm{mg}$ sample dissolved in $1 \mathrm{~mL}$ of $\mathrm{MeOH}$ and centrifuged. Volume of sample injection into HPLC system (1200 Series LC, Agilent Technologies, Wilmington, DE, USA) was $5 \mu \mathrm{L}$. $150 \mathrm{~cm} \times 2 \mathrm{~mm}^{2}, 4 \mu \mathrm{m}$ Synergi Hydro-RP $80 \AA$ column (Phenomenex, Torrance, CA, USA) was used for LC separation at $30{ }^{\circ} \mathrm{C}$. ESI activated at $3 \mathrm{kV}$ and $380{ }^{\circ} \mathrm{C}$ as a source temperature. LC-ESI-MS was measured under the following conditions: capillary voltage $=3 \mathrm{kV}$, cone voltage $=30 \mathrm{kV}$, source offset $=30 \mathrm{~V}$, nebulizer pressure $=15$ bar, desolvation gas flow-rate $=650 \mathrm{~L} / \mathrm{h}$, cone gas flow-rate $=150 \mathrm{~L} / \mathrm{h}$, fragmentor voltage $=90 \mathrm{~V}$, collision voltage $=20 \mathrm{~V} .0 .1 \%$ formic acid in distilled water as mobile phase $\mathrm{A}$ and $0.1 \%$ formic acid in acetonitrile as mobile phase B separated the sample and went into the ESI chamber at a flow rate of $0.5 \mathrm{~mL} / \mathrm{min}$ for $20 \mathrm{~min}$. Sample was detected by multiple-reaction monitoring mode (MRM) of monitoring the transition pairs at $\mathrm{m} / \mathrm{z} 252.1 / 136.1$.

\section{Cell culture}

The cisplatin sensitive ovarian cancer cell lines (CSC) A2780 and the cisplatin resistant cell lines (CRC) A2780cis were obtained from Dr. Jung-Hyuck Ahn (Ewha Womans University school of medicine, Seoul, Korea). A2780 and A2780cis cells were cultured in RPMI 1640 (Welgene, Daegu, South Korea) supplemented with 10\% fetal bovine serum (FBS) (Atlas, Fort Collins, CO, USA), $1 \%$ penicillin/streptomycine (Gibco, Gaithersberg, MD, USA) in a humidified atmosphere of $5 \% \mathrm{CO}_{2}$ at $37{ }^{\circ} \mathrm{C}$. A2780cis cells were supplemented $100 \mu \mathrm{M}$ of cisplatin (sigma, St. Louis, MO, USA) in medium every even cell passage. To investigate anti-cancer effects of $\mathrm{SbE}$, cells were cultured in RPMI 1640 supplemented with 10\% FBS and $1 \%$ penicillin/streptomycine. After $24 \mathrm{~h}$, $100 \sim 400 \mu \mathrm{g} / \mathrm{mL}$ of $\mathrm{SbE}$ and/or $10 \sim 100 \mu \mathrm{M}$ of cisplatin or $28 \sim 56 \mu \mathrm{M}$ of baicalin diluted in serum free RPMI 1640 were treated the cells for $24 \mathrm{~h}$.

\section{MTT assays}

Cell viability was measured by MTT assays [24]. 1X10 ${ }^{4}$ cells per well were seeded in 96-well plates and 
incubated at $37{ }^{\circ} \mathrm{C}$. After $24 \mathrm{~h}$, a range of concentrations of $\mathrm{SbE}$ and/or cisplatin or baicalin were treated to wells and incubated at $37^{\circ} \mathrm{C}$ for $24 \mathrm{~h}$. After $22 \mathrm{~h}$ treatment of $\mathrm{SbE}$ and/or cisplatin or baicalin, 3-[4,5-dimethylthiazol2-yl]-2,5-diphenyltetrazoliumbromide (MTT) (Sigma Aldrich, St, Louis MO, USA) solution like one-tenth the original culture volume was added in the treated cells for $2 \mathrm{~h}$. MTT solution was dissolved in phosphate buffered saline (PBS) to make stock solution $5 \mathrm{mg} / \mathrm{mL}$. Then DMSO was added to convert MTT to purple formazan in mitochondria of viable cells. Microplate reader (Biochrom, Berlin, Germany) read viable cells using the absorbance of $562 \mathrm{~nm}$.

\section{RNA isolation and reverse transcription}

A2780 and A2780cis cells were harvested after treated with various concentrations of $\mathrm{SbE}$ and/or cisplatin or baicalin for $24 \mathrm{~h}$. Total RNA was extracted using the trizol reagent (Life Technologies, Gaithersburg, MD, USA) and isopropanol precipitation. The pellets were dissolved in Tris-EDTA (TE) buffer. 500 ng RNA was used for complementary DNA (cDNA) synthesis using RevertAid reverse transcriptase (Thermo Scientific, Waltham, MA, USA), according to the manufacturer's protocol.

\section{Quantitative reverse transcriptase (qRT)-PCR}

CDNA was used for qRT-PCR to investigate gene expression levels. qRT-PCR was performed with SYBR Green PCR Master mix (Qiagen, Hilden, Germany) using PCR machine, Rotor-Gene Q machine (Qiagen, Hilden, Germany). The primer pairs were the followings: for Atg5, 5'-TGGAGTAGGTTTGGCTTTGG-3' and 5' - A TGGTTCTGCTTCCCTTTCA-3', for Atg12, 5' -CCT TTGCTCCTTCCCCAGA-3' and 5'-ATCCCCACGCC TGAGACTT-3', for Bax, 5'-CGTGGACACAGACTC CCC-3' and ' 5' -CCAATGTCCAGCCCATGATG-3', for Beclin 1, 5'-ACCAACGTCTTTAATGCAACCT-3' and 5' - CATGGAGCAGCAACACAGTC-3', for DRAM, 5' CATCCCCATGATTGTCTGTG-3' and 5'-AAAGGCC ACTGTCCATTCAC-3', for HDAC1, 5'- GGTCTC TACCGAAAAATGGAAA-3' and $5^{\prime}$-TTGCTGTACTC CGACATGTTATC-3', for $p 21,5^{\prime}$ '-TGTCTTGTACCC TTGTGCCT-3' and '5'- GGCGTTTGGAGTGGTA GAAA-3', for $p 53,5^{\prime}$-GCTGCTCAGATAGCGATGGT$3^{\prime}$ and $5^{\prime}$-CACGCACCTCAAAGCTGTTC-3', and for TBP, 5' -AGCCAAGAGTGAAGAACAGTCC-3' and 5' CACAGCTCCCCACCATATTC-3'. Amplification was done at $95{ }^{\circ} \mathrm{C}$ for $5 \mathrm{~min}$, followed by 40 cycles at $95{ }^{\circ} \mathrm{C}$ for $5 \mathrm{~s}$ and at $60{ }^{\circ} \mathrm{C}$ for $10 \mathrm{~s}$. The relative expression of each gene of interest was calculated by normalization against TATA-box binding protein (TBP) expression levels in each sample.

\section{Statistical analysis}

Results of cell viability and mRNA expression levels were indicated as mean \pm standard deviation. The results were analyzed using two tailed Student's t-test using Microsoft Excel 2010 (Microsoft, Redmond, WA, USA) and one-way analysis of variance (ANOVA) followed by Duncan post hoc test using SAS 9.4 (SAS Inc., Cary, NC, USA). $P<0.05$ was considered to indicate a statistical significance in all experiments.

\section{Results}

Ovarian cancer cell models differently respond to cisplatin and cisplatin treatment induces p53-dependent apoptosis in CSC, not in CRC

To investigate the mechanisms underlying cisplatin resistance, we used a pair of ovarian cancer cell lines; the cisplatin sensitive A2780 as CSC and its counterpart that acquires the resistance as CRC. We firstly confirmed that CRC was less sensitive to cisplatin, compared to $\mathrm{CSC}$. The response to cisplatin was measured by cell viability using an MTT assay. CSC and CRC were treated with from $10 \mu \mathrm{M}$ to $100 \mu \mathrm{M}$ of cisplatin for $24 \mathrm{~h}$. As shown in Fig. 1a, cisplatin treatment decreased cell viability and the response to cisplatin in CRC was significantly lower than that in CSC. Cell viability in CRC was significantly higher than it in CSC in $10 \mu \mathrm{M}, 30 \mu \mathrm{M}$, and $100 \mu \mathrm{M}$ of cisplatin-treated groups $(p<0.001)$. Cell viabilites in CSC decreased to $84 \%, 47 \%$, and $13 \%$ by $10 \mu \mathrm{M}, 30 \mu \mathrm{M}$, and $100 \mu \mathrm{M}$ of cisplatin treatment, respectively, compared with the non-treated group, while those in CRC decreased to $110 \%, 91 \%$, and $33 \%$. The difference of response to cisplatin between CSC and CRC was greater in the $30 \mu \mathrm{M}$ of cisplatin-treated group than in the $10 \mu \mathrm{M}$ or $100 \mu \mathrm{M}$ of cisplatin-treated groups. Therefore, we used $30 \mu \mathrm{M}$ of cisplatin treatment for further experiments.

We examined whether the difference in the induction of p53-mediated apoptotic pathway could explain the cisplatin resistance-involving mechanism in CRC. Expression levels of $p 53, p 21, B a x$, and DRAM genes were measured in the cells treated with $30 \mu \mathrm{M}$ of cisplatin for $24 \mathrm{~h}$. As shown in Fig. 1b, mRNA expression levels of pro-apoptotic $p 53(p<0.05)$ and its target genes such as $p 21(p<0.001)$, and Bax $(p<0.05)$, and DRAM $(p<0.05)$ significantly increased in the cisplatin-treated group, compared with the non-treated group in CSC, indicating that the cell death of CSC with cisplatin treatment invovled apoptosis through the p53-dependent pathway. On the other hand, CRC did not show significant changes in the expression of $p 53$ and Bax (Fig. 1c). Although mRNA levels of $p 21$ significantly increased, expression of DRAM rather decreased upon cisplatin treatment $(p<0.001$; Fig. $1 \mathrm{c})$. The results suggested that cisplitin treatment failed to induce apoptosis in the 

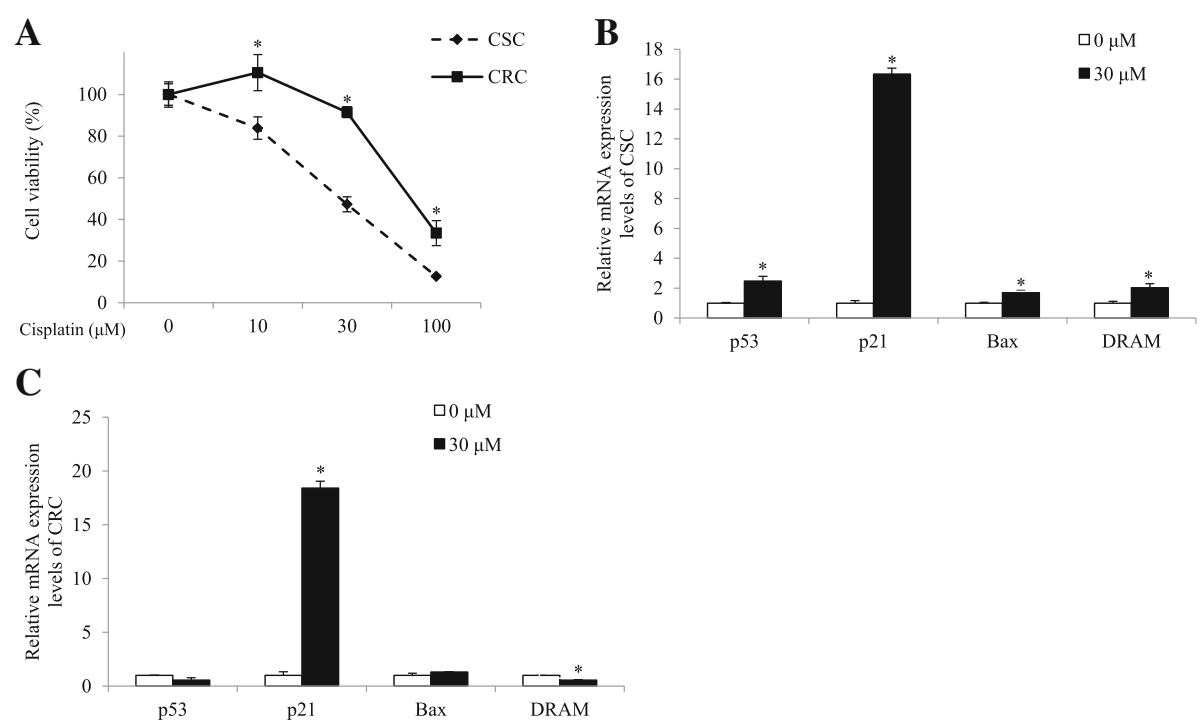

Fig. 1 Effects of cisplatin on the cell viability and mRNA expression levels of p53, p21, Bax, and DRAM in CSC and CRC. Cells were exposed $10 \sim 100 \mu \mathrm{M}$ of cisplatin for $24 \mathrm{~h}$. a Cell viability according to cisplatin treatment in CSC and CRC was measured by MTT assay. After cells were exposed $30 \mu \mathrm{M}$ of cisplatin for $24 \mathrm{~h}$, mRNA expression levels of (b). p53, p21, Bax, and DRAM in CSC and (c). p53, p21, Bax, and DRAM in CRC were quantified by qPCR. All mRNA expression levels were normalized against TBP. Each value is the mean \pm SD. $p<0.05$ was taken to define statistical significance. Two tailed Student's t-test and one-way ANOVA followed by Duncan post hoc test

CRC, unlike CSC, due to the decreased expression level of DRAM gene, which is required for activation of the p53-dependent pathway.

\section{Cisplatin-induced cell death is not mediated by autophagy in ovarian cancer models}

We investigated whether cisplatin-induced cell death is also mediated by autophagy. CSC and CRC were treated with $30 \mu \mathrm{M}$ of cisplatin for $24 \mathrm{~h}$ and mRNA levels of autophay genes, Beclin 1, Atg12, and Atg5 genes were measured. In CSC, mRNA expression levels of Beclin 1 and Atg5 did not significantly alter or decreased, respectively, while those of Atg12 increased in cisplatin-treated group compared with the non-treated group $(p<0.01$; Fig. 2a). Similarily, in CRC, mRNA expression levels of Beclin 1 and Atg5 decreased, while those of Atg12 increased upon cisplatin treatment $(p<0.01$; Fig. $2 \mathrm{~b})$. The results suggest cisplatin-induced cell death in CSC is not mediated by autophagy, since Atg5 induction, which is esstial for the conjugation with Atg12 in autophagy, was not complete. In CRC, neither the p53-mediated apoptosis nor autophagy did not taken place, indicating the resistance against cisplatin.

\section{SbE is more effective to decrease cell viability than baicalin both in CSC and CRC}

Next, we investigated whether SbE induced cell death in CSC and CRC to test a potential of SbE as an anticancer agent. We first analyzed quantitatively baicalin content in SbE using LC-MS analysis. The baicalin is a major flavonoid in $\mathrm{SbE}$ and has been reported for its effects on various cancer cells to inhibit cell proliferation and to induce cell death [21]. The quantity of baicalin in SbE was measured (Fig. 3a) and calculated according to a standard curve (Fig. $3 \mathrm{~b}$ ). As a result, we found that the content of baicalin in SbE was $74 \mathrm{mg} / \mathrm{g}$ (7.4\%). Next, we investigated whether $\mathrm{SbE}$ and baicalin induced cell death in CSC and CRC at the various concentrations using an MTT assay. First, the cell lines were treated with from $200 \mu \mathrm{g} / \mathrm{mL}$ to $400 \mu \mathrm{g} / \mathrm{mL}$ of $\mathrm{SbE}$ and from $28 \mu \mathrm{M}$ to $56 \mu \mathrm{M}$ of baicalin, i.e. equivalent to the amounts in the extract, for $24 \mathrm{~h}$ to compre the effect of the extract and the single compound. As shown in Fig. 3c and d, both $\mathrm{SbE}$ and baicalin treatment decreased cell viability, however, the response of the $\mathrm{SbE}$ was significantly greater than that of baicalin. In CSC, cell viability in $200 \mu \mathrm{g} / \mathrm{mL}$ and $400 \mu \mathrm{g} / \mathrm{mL}$ of SbE-treated groups were significantly lower than it in $28 \mu \mathrm{M}$ and $56 \mu \mathrm{M}$ of baicalin-treated groups, respectively $(p<0.01$ and $p<0.001$, respectively). Likewise, in CRC, $200 \mu \mathrm{g} / \mathrm{mL}$ and $400 \mu \mathrm{g} / \mathrm{mL}$ of SbE treatment were significantly decreased compared to $28 \mu \mathrm{M}$ and $56 \mu \mathrm{M}$ of baicalin treatment, respectively ( $p<0.05$ and $p<0.01$, respectively). Cell viability in CSC decreased to $53 \%$ and $33 \%$ by $200 \mu \mathrm{g} / \mathrm{mL}$ and $400 \mu \mathrm{g} / \mathrm{mL}$ of $\mathrm{SbE}$ treatment, respectively, compared with the non-treated group (Fig. 3c), while thoes in CRC decreased to $71 \%$ and $55 \%$ (Fig. 3d). On the other hand, cell viability in CSC decreased to $77 \%$ and $67 \%$ by $28 \mu \mathrm{M}$ and $56 \mu \mathrm{M}$ of baicalin treatment, respectively (Fig. 3c), while those in CRC decreased to $86 \%$ and $80 \%$ 


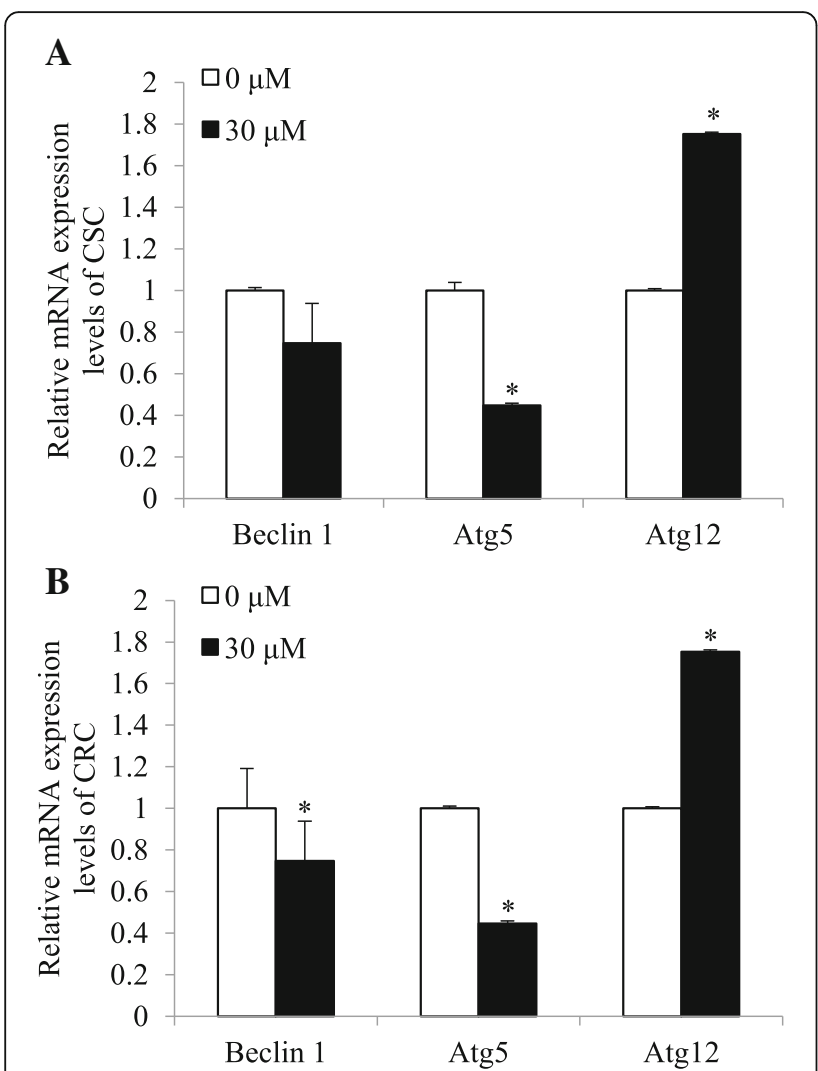

Fig. 2 Effects of cisplatin on mRNA expression levels of Beclin 1, Atg5, and Atg 12 in CSC and CRC. After cells were exposed $30 \mu \mathrm{M}$ of cisplatin for 24 h, mRNA expression levels of (a). Beclin 1, Atg5, and Atg12 in CSC and (b). Beclin 1, Atg5, and Atg12 in CRC were quantified by qPCR. All mRNA expression levels were normalized against TBP. Each value is the mean $\pm \mathrm{SD}$. $p<0.05$ was taken to define statistical significance. One-way ANOVA followed by Duncan post hoc test

(Fig. 3d). Because the effects on cell viability of the extract is greater than that of single compound, baicalin, we focused on the effect of SbE for a subsequent experiments. We tested the effect of SbE on cell viability in more various concentrations. It effectively decreased cell viability in a dose-dependent manner in CSC and CRC and the response to $\mathrm{SbE}$ in CRC was significantly lower than in CSC (Fig. 3e).

\section{SbE treatment induces p53-dependent apoptosis in CSC, not in CRC}

We investigated by which mechanism SbE induced the cell death. Firstly, we tested whether SbE induced p53mediated apoptotic pathway and its effect was similar in CSC and CRC. Expression level of p53-mediated apoptotic pathway such as $p 53, p 21, B a x$, and DRAM genes were measured in the cells treated with $200 \sim 400 \mu \mathrm{g} /$ $\mathrm{mL}$ of SbE for $24 \mathrm{~h}$. In CSC, mRNA expression levels of p53 ( $p<0.01), p 21(p<0.05), \operatorname{Bax}(p<0.05)$, and DRAM $(p<0.05)$ significantly increased in SbE-treated group, compared with the non-treated group (Fig. 4a).
On the contrary, CRC showed that mRNA expression levels of $p 53$ and DRAM decreased $(p<0.001)$ and those of $p 21$ and $B a x$ did not significantly alter after $\mathrm{SbE}$ treatment (Fig. 4b). The data suggested that SbE treatment induced apoptosis in the CSC, at least in part, via p53 pathway, but not in the CSC.

\section{SbE treatment induces autophagy in CSC, not in CRC}

We investigated whether SbE-induced cell death also involved the autophagy mechanism. Expression levels of autophagy genes, Beclin 1, Atg12, and Atg5 were measured in the cells treated with $200 \sim 400 \mu \mathrm{g} / \mathrm{mL}$ of SbE for $24 \mathrm{~h}$. In CSC, mRNA expression levels of Beclin 1 did not significantly alter, while those of Atg5 $(p<0.05)$ and $\operatorname{Atg} 12(p<0.01)$ significantly increased in the SbEtreated group compared with the non-treated group (Fig. 5a). CRC, in contrast to CSC, showed that mRNA expression levels of Beclin 1 and Atg5 decreased $(p<0.05)$ after SbE treatment, while those of Atg12 increased $(p<0.001)$ in the SbE-treated group compared with the non-treated group (Fig. 5b). As similar to the response to cisplatin, the autophagy-mediated cell death was induced in CSC, but not in CRC. The results proposed that SbE could not induce cell death neither p53mediated apoptotic pathway or autophagy and its mechanism is involved in the resistance in CRC.

\section{CSC and CRC similarly respond to SbE combined with cisplatin}

Next, we tested whether a combined treatment of SbE and cisplatin had additive or synergic effects in CSC and CRC. CSC and CRC were treated with $200 \sim 400 \mu \mathrm{g} / \mathrm{mL}$ of $\mathrm{SbE}$ and $10 \mu \mathrm{M} \sim 100 \mu \mathrm{M}$ of cisplatin for $24 \mathrm{~h}$. The response to the combined treatment in CRC was as sensitive as that in CSC by using MTT assays (Fig. 6). When the ovarian cancer cells were treated with the combined treatment, the cell viability between CSC and CRC gradually became closer depending on the concentration of the $\mathrm{SbE}$ at $30 \mathrm{uM}$ of cisplatin, where the viability showed maximal difference between CSC and CRC upon only cisplatin treatment (Fig. 1a). The cell viability in CSC decreased to $47 \%, 43 \%$, and $14 \%$, while those in CRC decreased to $91 \%, 64 \%$, and $16 \%$ by $0 \mu \mathrm{g} / \mathrm{mL}, 200 \mu \mathrm{g} / \mathrm{mL}$, and $400 \mu \mathrm{g} / \mathrm{mL}$ of $\mathrm{SbE}$ with $30 \mu \mathrm{M}$ of cisplatin compared with the non-treated group (Fig. 6b). The data show that the combined treatment of $\mathrm{SbE}$ and cisplatin has a potential as a chemotherapeutic method to overcome chemoresistance.

The treatment of $\mathrm{SbE}$, combined with cisplatin, induces not apoptotic but autophagic cell death in CRC

We further examined a possible mechanism underlying the combination effect. We tested whether the cell death upon the $\mathrm{SbE}$ treatment combined with cisplatin is 


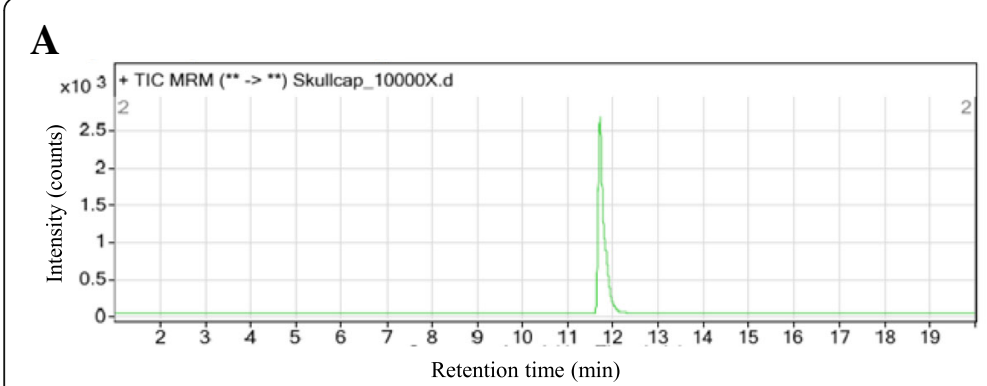

B

C

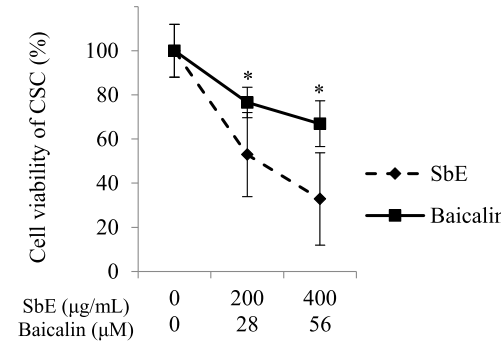

$\mathrm{D}$

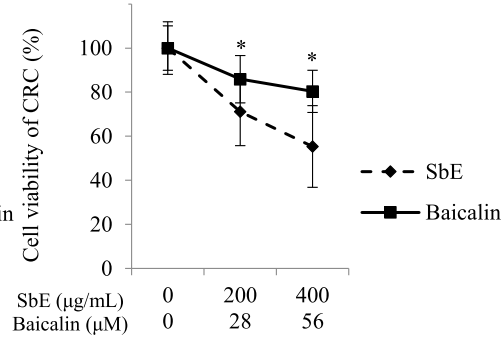

$\mathbf{E}$
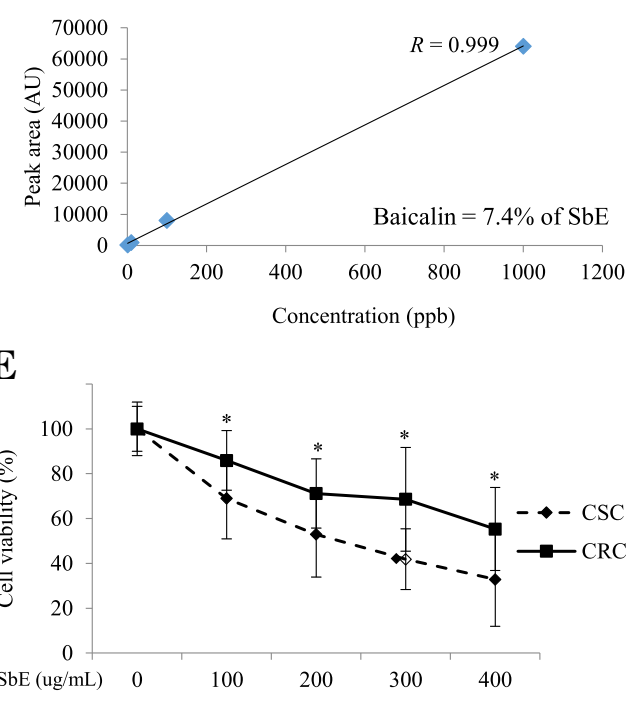

Fig. 3 Quantitative analysis of baicalin in SbE and effects of SbE and baicalin on the cell viability in CSC and CRC. a LC-MS analysis of baicalin in SbE. b Baicalin peak area in LC-MS as a function of baicalin concentration (correlation coefficient, $R=0.999$ ) and the percent content of baicalin in SbE. Cells were exposed $200 \sim 400 \mu \mathrm{g} / \mathrm{mL}$ of SbE or $28 \sim 56 \mu \mathrm{M}$ of baicalin for $24 \mathrm{~h}$. Cell viability according to SbE or baicalin treatment in (c) CSC and (d) CRC was determined by MTT assay. Cells were exposed $100 \sim 400 \mu \mathrm{g} / \mathrm{mL}$ of SbE for $24 \mathrm{~h}$. e Cell viability according to various concentrations of SbE treatment in CSC and CRC was determined by MTT assay. Each value is the mean \pm SD. $p<0.05$ was taken to define statistical significance. Two tailed Student's t-test

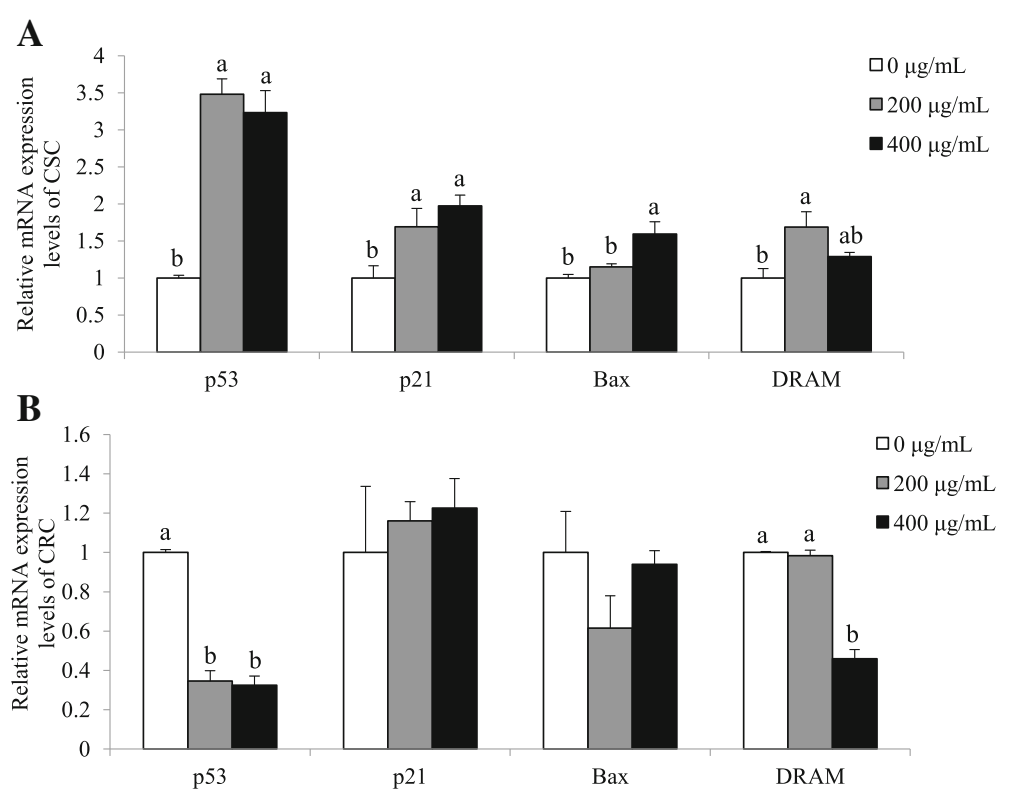

Fig. 4 Effects of SbE on mRNA expression levels of p53, p21, Bax, and DRAM in CSC and CRC. After cells were exposed $200 \sim 400 \mu \mathrm{g} / \mathrm{mL}$ of SbE for $24 \mathrm{~h}$, mRNA expression levels of (a). p53, p21, Bax, and DRAM in CSC and (b) p53, p21, Bax, and DRAM in CRC were quantified by qPCR. All mRNA expression levels were normalized against TBP. Each value is the mean \pm SD. $p<0.05$ was taken to define statistical significance. One-way ANOVA followed by Duncan post hoc test 


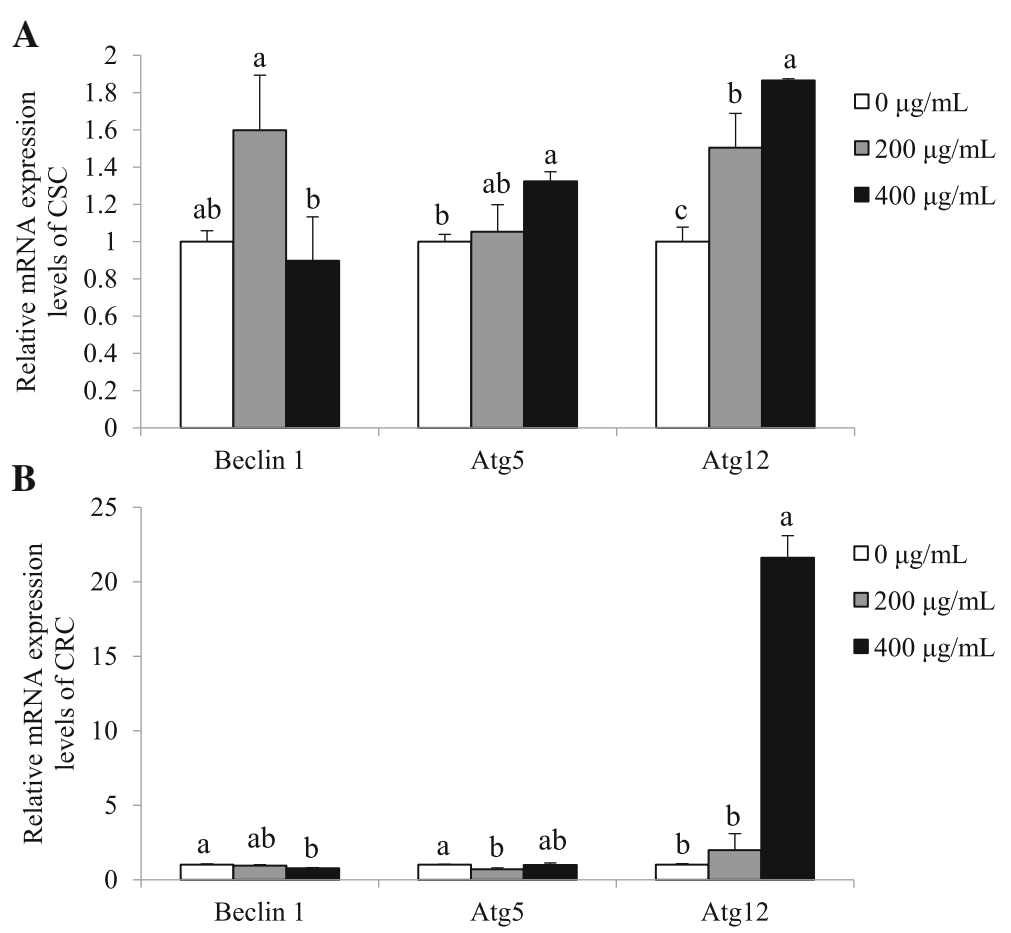

Fig. 5 Effects of of SbE on mRNA expression levels of Beclin 1, Atg5, and Atg12 in CSC and CRC. After cells were exposed $200 \sim 400 \mu \mathrm{g} / \mathrm{mL}$ of SbE for 24 h, mRNA expression levels of (a) Beclin 1, Atg5, and Atg12 in CSC and (b) Beclin 1, Atg5, and Atg12 in CRC were quantified by qPCR. All mRNA expression levels were normalized against TBP. Each value is the mean \pm SD. $p<0.05$ was taken to define statistical significance. One-way ANOVA followed by Duncan post hoc test

mediated by either $\mathrm{p} 53$ pathway and/or autophagic pathway in the condition of $200 \sim 400 \mu \mathrm{g} / \mathrm{mL}$ of $\mathrm{SbE}$ and $30 \mu \mathrm{M}$ of cisplatin treatment for $24 \mathrm{~h}$. In CRC, mRNA expression level of $p 53$ significantly increased in a SbE $(p<0.05)$, while that of $p 21$ decreased $(p<0.001$; Fig. 7a). In addition, the expression of $B a x$ and $D R A M$ did not significantly change (Fig. 7a). Surprisingly, the dramatically decreased expression of $p 21$ was not consistent with the increased expression of $p 53$, even though $\mathrm{p} 21$ is a well-known transcriptional target of $\mathrm{p} 53$. We investigated if the expression of $p 21$ was regulated by additional factors such as epigenetic modulators. HDAC1 and p53 have been demonstrated as antagonistic regulators at the $p 21$ locus. mRNA expression levels of $H D A C 1$ significantly increased upon the combined treatment of $\mathrm{SbE}$ and cisplatin $(p<0.05$; Fig. 7b), restulting in the transcriptional repression of $p 21$. Instead, we examined mRNA expression levels of autophagy genes such as Beclin 1, Atg5, and Atg12. mRNA expression levels of Beclin 1 did not change (Fig. 7c) in the combination-treated group, compared with the non-treated group. However, the expression of $\operatorname{Atg} 5$ and $\operatorname{Atg} 12$ increased upon the treatment $(p<0.05$; Fig. $7 \mathrm{c})$. Taken together, the results demonstrated that the combination therapy of
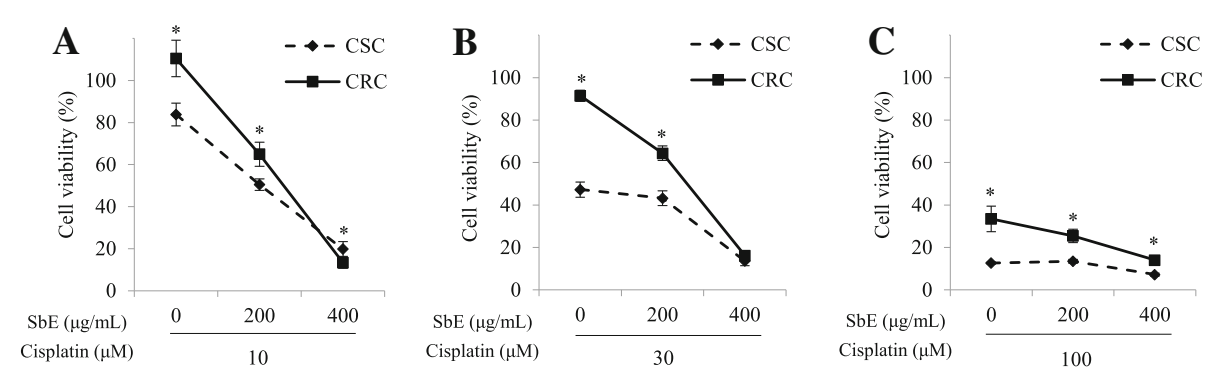

Fig. 6 Combination effects of SbE and cisplatin on the cell viability in CSC and CRC. After cells were exposed $200 \sim 400 \mu \mathrm{g} / \mathrm{mL}$ of SbE and $30 \mu \mathrm{M}$ of cisplatin for $24 \mathrm{~h}$. Cell viability according to the combination of SbE and (a) $10 \mu \mathrm{M}$, (b) $30 \mu \mathrm{M}$, and (c) $100 \mu \mathrm{M}$ of cisplatin treatment in CSC and CRC was measured by MTT assay. Each value is the mean \pm SD. $p<0.05$ was taken to define statistical significance. Two tailed Student's t-test 


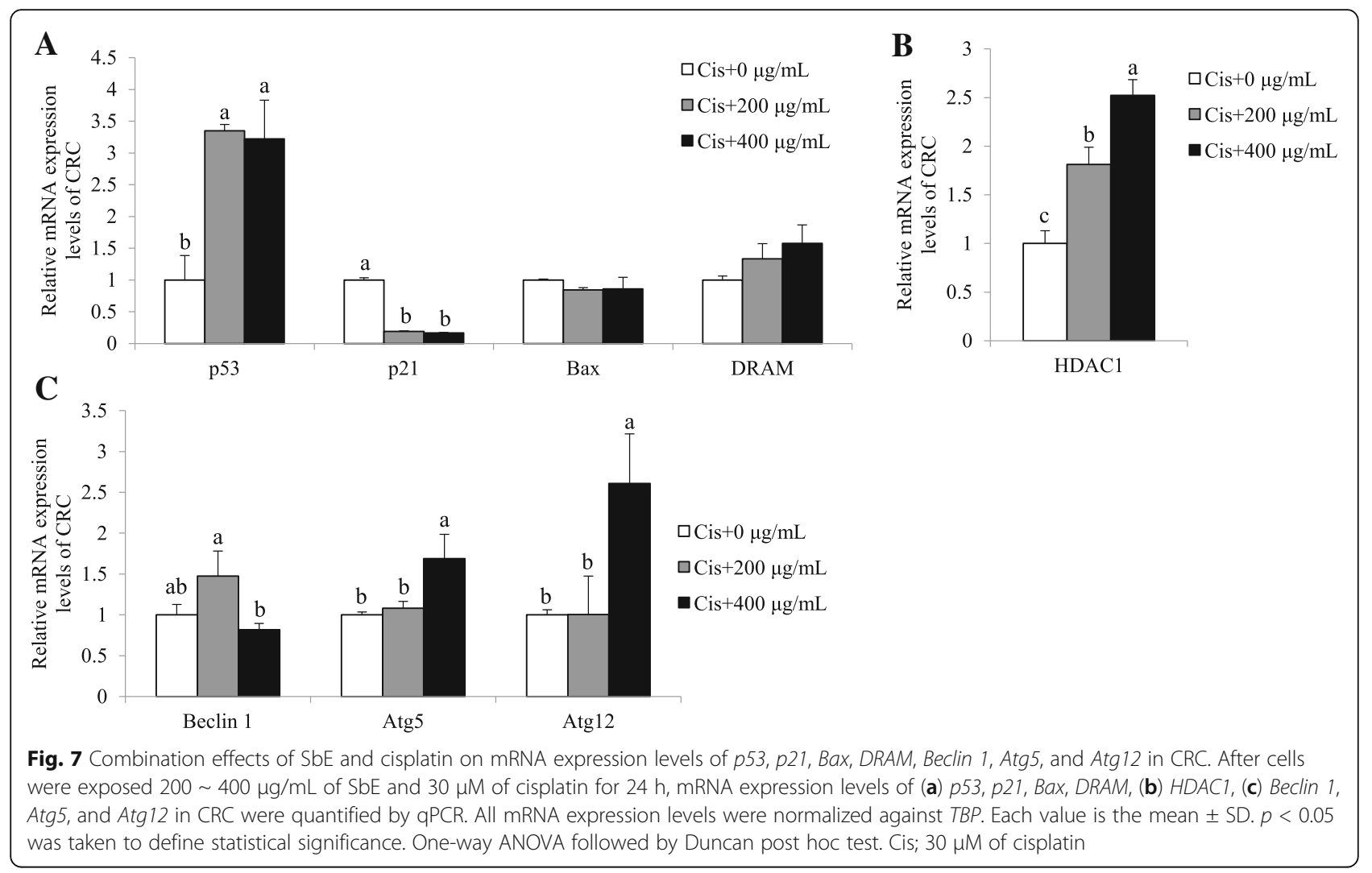

SbE and cisplatin effectively induced cell death even in CRC via the autophagy pathway independent of Beclin 1.

\section{Discussion}

In this study, we investigated the effect and the possible molecular mechanism of $\mathrm{SbE}$ on cisplatin resistance of ovarian cancer by three steps. First of all, we validated the cell models whether CSC and CRC showed different sensitivity against cisplatin, as expected. Next, we examined whether SbE induced cell death in ovarian cancer and what molecular mechanism was involved. Lastly, we demonstrated the effects of combined treatment with SbE and cisplatin on cisplatin resistance in ovarian cancer.

We found that cisplatin induced cell death in CSC in a dose-dependent manner based on up-regulation of genes associating apoptosis pathway such as $p 53, p 21$, and $B a x$ expression. As the cellular response to DNA damage, the tumor suppressor and transcription factor p53 and its targets, cyclin-dependent kinase inhibitor p21 and pro-apoptotic Bax, play important roles in apoptosis [25]. It is also critical to modulate the chemosensitivity of tumors by controlling cell death [26]. However, CRC showed cisplatin resistance even though the expression of pro-apoptotic protein $p 21$ was up-regulated. One possibility is due to decreased DRAM mRNA expression level. A previous study showed that DRAM is a major component of p53-induced apoptosis [16]. Its knockdown decreased percentage of apoptosis even though mRNA expression levels of $p 53$ and $p 21$ increased in TetOn-p53 cells and RKO cells. Our results demonstarated that cisplatin treatment led to down-regulated expression of the DRAM gene in CRC, while it did not in CSC. Therefore, p53-mediated apoptotic pathway seemed to be blocked due to the decreased expression of $D R A M$ in the cisplatin resistant cells.

The cisplatin-mediated cell death was not dependent on autophagy. The mRNA expression of Atg5 levels was significantly decreased in both CSC and CRC. Because Atg12-Atg5 conjugate is an essential factor of autophagy, concordant expression of Atg12 and Atg5 expression is induced during autophagy. In a previous study, the Atg 5 mutant was unable to generate the Atg12-Atg5 conjugate, resulting in the decrease of autophagic activity rapidly compared with the wild type [27].

Recently, the flavonoid baicalin enriched in herbal medicines including SbE [28] has reported to have anticancer properties in vivo and in vitro $[21,29]$. We showed that an inhibitory effect of $\mathrm{SbE}$ as the extract on cell viability was greater than that of baicalin as a single compound in ovarian cancer cells. SbE induced cell death, depending on its concentration, by inducing expression of apoptosis genes, such as $p 53, p 21$, and $B a x$, and autophagy genes such as $\operatorname{Atg} 5$ and $\operatorname{Atg} 12$ in CSC, 
but not in CRC. The results showed that CRC had lower efficiency against a single treatment of cisplatin or SbE, compared to CSC. However, combination treatment of $\mathrm{SbE}$ and cisplatin enhanced anti-cancer effects via induction of cell death. When $400 \mu \mathrm{g} / \mathrm{mL}$ of SbE and $30 \mu \mathrm{M}$ of cisplatin were treated at the same time, cell viability was no difference between CSC and CRC. Intriguingly, the combination treatment did not fully induce p53mediatic apoptotic pathway. Even though $p 53$ expression increased, its target gene $p 21$ expression decreased. One of the possibilities is the up-regulation of HDAC1, an epigenetic modulator. Recently, epigenetic mechanism has been highlighted in cancer field. Epigenetic modulation contributes to cancer development, progress, and treatment, since it regulates expression of oncogenes and tumor suppressor genes by altering chromatin structure [30]. Chemical modifications on DNA and core histones, the octamer of proteins wrapping DNA, make chromatin condensate or unwind without altering DNA sequence [31]. Histone acetylation, an example of histone modifications, is a process that an acetyl group is bound to histone resulting in neutralizing DNA charge then forming euchromatin. Euchromatic structure allows a gene to be up-regulated by loosely unfolding nucleosomes and making transcriptional factors easily access to DNA [32]. The acetylated histones are accomplished by histone acetyltransferases (HATs) and the acetyl group is detached by histone deacetylases (HDACs) from histones. A previous study showed that p53 and the epigenetic regulator $\mathrm{HDAC} 1$ are antagonistic regulators of the p21 [33]. p53 transcriptionally activates p21 through binding to the transcription factor $\mathrm{Sp} 1$ in the activation of the p21 promoter. On the other hand, HDAC1 transcriptionally represses $p 21$ gene expression by blocking the interaction between p53 and Sp1. In CRC, the combination treatment repressed $p 21$ gene expression through HDAC1 activation, resulting in inactivation of apoptosis. However, further experiments are needed to verify whether up-regulation of $H D A C 1$ expression resulted from the combined treatment alters histone acetylation levels, in particular at the $p 21$ locus. Nevertheless, the combination of SbE and cisplatin induced cell death via autophagy in $\mathrm{CRC}$, showing effectiveness to overcome the resistance. It was mediated by noncanonical Beclin 1-independent autophagic cell death based on the increase of Atg 5 and Atg12 expression, but no change in Beclin-1 expression. Beclin 1 is related to form a phagophore which is consist of autophagosome [18]. However, Beclin 1-independent autophagy has been reported [34, 35]. Resveratrol induced cell death through Beclin 1-independent Atg12-Atg5-dependent autophagy [34]. Arsenic trioxide also induced Beclin 1-independent autophagic pathway in ovarian cancer cells. In the arsenic trioxide-treated ovarian cancer cells, Atg5 knockdown reduced autophagy via altering the ratio of LC3-II/LC3-I, which is an indicator of the autophagic progress [36]. In contrast, Beclin 1 knockdown did not alter the ratio of LC3-II/LC3-I [35]. Our data suggest that the combination treatment of SbE and cisplatin produced synergistic anti-cancer effect even in cisplatin resistant ovarian cells. Further analysis is needed to confirm the molecular alterations at protein levels and the extension of anti-cancer effects, i.e. the percentage of apoptotic and/or autophagic cell death.

\section{Conclusions}

Compared with $\mathrm{SbE}$ or cisplatin alone, the combination treatment of $\mathrm{SbE}$ and cisplatin had strengthened anticancer effects in ovarian cancer cells. Although SbE induced cell death in ovarian cancer cells in a dosedependent manner, the efficiency was significantly lower in CRC, compared to CSC. However, the combination treatment with cisplatin led to the effect on CRC, as similar as on CSC, suggesting the effectiveness of the combined treatment over chemoresistance. The combination treatment in CRC induced autophagy by upregulated expression of $\operatorname{Atg} 5$ and Atg12. It was different from the fact that $\mathrm{SbE}$ as a single treatment failed to induce apoptosis via p53 or autophagic pathways in CRC. Taken together, the results demonstrated that the combination treatment of $\mathrm{SbE}$ and cisplatin had a synergistic effect by inducing Beclin 1-independent autophagy in CRC. The findings suggest that the combination of SbE and cisplatin may be useful for a potential chemotherapy to treat ovarian cancer.

\section{Abbreviations \\ 3 MTT: 3-[4,5-dimethylthiazol-2-yl]-2,5-diphenyltetrazoliumbromide; Atg: Autophagy-related protein; CRC: Cisplatin resistant human ovarian cancer cell line; CSC: Cisplatin sensitive human ovarian cancer cell line; DMSO: Dimethyl sulfoxide; DRAM: Damage-regulated autophagy modulator; FBS: Fetal bovine serum; HATs: Histone acetyltransferases; HDACs: Histone deacetylases; LC3: Microtubule-associated protein light chain; SbE: Extract of Scutellaria baicalensis}

\section{Acknowledgements}

There is no contributor who does not meet the criteria for authorship.

\section{Funding}

This study was supported by the National Research Foundation of Korea (NRF 2014R1A1A2058942 and 2016R1D1A1A02937546). BYC and YKL were supported by Brain Korea 21 plus project (22A20130012143).

\section{Availability of data and materials \\ The datasets supporting the conclusions of this article are included within the article.}

\section{Author's contributions}

SJP and YJP designed hypothesis and supervise experiments; JCJ and SJP provided the SbE; BYC, YKL and IJ performed the experiments; BYC and YJP analyzed the data, and wrote the manuscript. All authors read and approved the final manuscript.

Competing interests

The authors declare that they have no competing interests. 


\section{Consent for publication}

Not applicable.

\section{Ethics approval and consent to participate}

Not applicable.

\section{Publisher's Note}

Springer Nature remains neutral with regard to jurisdictional claims in published maps and institutional affiliations.

\section{Author details}

${ }^{1}$ Department of Nutritional Science and Food Management, College of Science \& Industry Convergence, Ewha Womans University, Seoul, Republic of Korea. ${ }^{2}$ Department of Sasang Constitutional Medicine, College of Korean Medicine, Wonkwang University, Iksan, Republic of Korea. ${ }^{3}$ Department of Bioconvergence, Korea basic science institute, Daejeon, Republic of Korea. ${ }^{4}$ Department of Sasang Constitutional Medicine, College of Korean Medicine, Woosuk University, Jeonju, Republic of Korea.

Received: 22 December 2016 Accepted: 8 May 2017

Published online: 25 May 2017

\section{References}

1. Torre LA, et al. Global cancer statistics, 2012. CA Cancer J Clin. 2015;65(2):87-108.

2. McGuire WP, et al. Cyclophosphamide and cisplatin compared with paclitaxel and cisplatin in patients with stage III and stage IV ovarian cancer. N Engl J Med. 1996;334(1):1-6.

3. Howlader N, Noone A, Krapcho M. SEER Cancer Statistics Review, 19752013. Bethesda: National Cancer Institute; 2016.

4. Markman M, et al. Responses to second-line cisplatin-based intraperitonea therapy in ovarian cancer: influence of a prior response to intravenous cisplatin. J Clin Oncol. 1991;9(10):1801-5.

5. Ohmichi $M$, et al. Mechanisms of platinum drug resistance. Trends Pharmacol Sci. 2005:26(3):113-6.

6. $\mathrm{Xu} \mathrm{X}$, et al. Genetic polymorphism of copper transporter protein 1 is related to platinum resistance in Chinese non-small cell lung carcinoma patients. Clin Exp Pharmacol Physiol. 2012;39(9):786-92.

7. Kelland LR. New platinum antitumor complexes. Crit Rev Oncol Hematol. 1993;15(3):191-219.

8. Dabholkar M, et al. ERCC1 and ERCC2 expression in malignant tissues from ovarian cancer patients. J Natl Cancer Inst. 1992;84(19):1512-7.

9. Sui $\mathrm{L}$, et al. Survivin expression and its correlation with cell proliferation and prognosis in epithelial ovarian tumors. Int J Oncol. 2002;21(2):315-20.

10. Perego $P$, et al. Association between cisplatin resistance and mutation of p53 gene and reduced bax expression in ovarian carcinoma cell systems. Cancer Res. 1996;56(3):556-62.

11. Green DR. Means to an end: apoptosis and other cell death mechanisms. Cold Spring Harbor: Cold Spring Harbor Laboratory Press; 2011.

12. Lowe SW, et al. p53-dependent apoptosis modulates the cytotoxicity of anticancer agents. Cell. 1993;74(6):957-67.

13. Chiocca EA. Oncolytic viruses. Nat Rev Cancer. 2002;2(12):938-50.

14. El-Deiry WS, et al. WAF1, a potential mediator of p53 tumor suppression. Cell. 1993;75(4):817-25.

15. Ahmad N, et al. Resveratrol causes WAF-1/p 21-mediated G1-phase arrest of cell cycle and induction of apoptosis in human epidermoid carcinoma A431 cells. Clin Cancer Res. 2001;7(5):1466-73.

16. Crighton D, et al. DRAM, a p53-induced modulator of autophagy, is critical for apoptosis. Cell. 2006;126(1):121-34.

17. Kondo $Y$, et al. The role of autophagy in cancer development and response to therapy. Nat Rev Cancer. 2005;5(9):726-34

18. Kaur J, Debnath J. Autophagy at the crossroads of catabolism and anabolism. Nat Rev Mol Cell Biol. 2015;16(8):461-72.

19. Zhang X-D, et al. DRAM1 regulates autophagy flux through lysosomes. PLoS One. 2013;8(5):e63245.

20. Huang W-H, Lee A-R, Yang C-H. Antioxidative and anti-inflammatory activities of polyhydroxyflavonoids of Scutellaria Baicalensis GEORGI. Biosci Biotechnol Biochem. 2006:70(10):2371-80.

21. Zhou Q-M, et al. The combination of baicalin and baicalein enhances apoptosis via the ERK/p38 MAPK pathway in human breast cancer cells. Acta Pharmacol Sin. 2009;30(12):1648-58.
22. Ikezoe T, et al. Baicalin is a major component of PC-SPES which inhibits the proliferation of human cancer cells via apoptosis and cell cycle arrest. Prostate. 2001;49(4):285-92.

23. Kumagai T, et al. Scutellaria Baicalensis, a herbal medicine: anti-proliferative and apoptotic activity against acute lymphocytic leukemia, lymphoma and myeloma cell lines. Leuk Res. 2007;31(4):523-30.

24. Mosmann T. Rapid colorimetric assay for cellular growth and survival: application to proliferation and cytotoxicity assays. J Immunol Methods. 1983;65(1-2):55-63.

25. Culmsee C, Mattson MP. p53 in neuronal apoptosis. Biochem Biophys Res Commun. 2005:331(3):761-77.

26. Harris CC. Structure and function of the p53 tumor suppressor gene: clues for rational cancer therapeutic strategies. J Natl Cancer Inst. 1996;88(20): 1442-55.

27. Mizushima $\mathrm{N}$, et al. A protein conjugation system essential for autophagy. Nature. 1998;395(6700):395-8.

28. Ye F, et al. Quality evaluation of commercial extracts of Scutellaria Baicalensis. Nutr Cancer. 2004:49(2):217-22.

29. Xu X-F, et al. Baicalin induces human mucoepidermoid carcinoma Mc3 cells apoptosis in vitro and in vivo. Investig New Drugs. 2011;29(4):637-45.

30. Egger $\mathrm{G}$, et al. Epigenetics in human disease and prospects for epigenetic therapy. Nature. 2004:429(6990):457-63.

31. Holliday R. Epigenetics: a historical overview. Epigenetics. 2006;1(2):76-80.

32. Jenuwein T, Allis CD. Translating the histone code. Science. 2001;293(5532): 1074-80.

33. Lagger $\mathrm{G}$, et al. The tumor suppressor p53 and histone deacetylase 1 are antagonistic regulators of the cyclin-dependent kinase inhibitor p21/WAF1/ CIP1 gene. Mol Cell Biol. 2003;23(8):2669-79.

34. Scarlatti F, et al. Role of non-canonical Beclin 1-independent autophagy in cell death induced by resveratrol in human breast cancer cells. Cell Death \& Differentiation. 2008:15(8):1318-29.

35. Smith $D$, et al. Arsenic trioxide induces a beclin-1-independent autophagic pathway via modulation of SnoN/SkiL expression in ovarian carcinoma cells. Cell Death \& Differentiation. 2010;17(12):1867-81.

36. Wu J, et al. Molecular cloning and characterization of rat LC3A and LC3B-two novel markers of autophagosome. Biochem Biophys Res Commun. 2006:339(1):437-42.

\section{Submit your next manuscript to BioMed Central and we will help you at every step:}

- We accept pre-submission inquiries

- Our selector tool helps you to find the most relevant journal

- We provide round the clock customer support

- Convenient online submission

- Thorough peer review

- Inclusion in PubMed and all major indexing services

- Maximum visibility for your research

Submit your manuscript at www.biomedcentral.com/submit
Biomed Central 